\title{
BMJ Open Recovery, rehabilitation and follow-up services following critical illness: an updated UK national cross-sectional survey and progress report
}

\author{
Bronwen Connolly (10 , ${ }^{1,2,3,4}$ Rhian Milton-Cole ${ }^{2}$ Claire Adams, ${ }^{5}$ Ceri Battle, ${ }^{6}$ \\ Joanne McPeake (D) ,7,8,9 Tara Quasim, ${ }^{7,8}$ Jon Silversides, ${ }^{10}$ Andrew Slack, ${ }^{11}$ \\ Carl Waldmann, ${ }^{12}$ Elizabeth Wilson, ${ }^{13}$ Joel Meyer, ${ }^{11}$ on behalf of The Faculty of \\ Intensive Care Medicine Life After Critical Illness Working Group
}

To cite: Connolly B, MiltonCole R, Adams C, et al. Recovery, rehabilitation and follow-up services following critical illness: an updated UK national cross-sectional survey and progress report. BMJ Open 2021;11:e052214. doi:10.1136/ bmjopen-2021-052214

- Prepublication history and additional supplemental material for this paper are available online. To view these files, please visit the journal online (http://dx.doi.org/10.1136/ bmjopen-2021-052214).

Received 11 April 2021 Accepted 31 August 2021

Check for updates

(C) Author(s) (or their employer(s)) 2021. Re-use permitted under CC BY-NC. No commercial re-use. See rights and permissions. Published by BMJ.

For numbered affiliations see end of article.

Correspondence to Dr Bronwen Connolly; B.Connolly@qub.ac.uk

\section{ABSTRACT}

Objective To comprehensively update and survey the current provision of recovery, rehabilitation and follow-up services for adult critical care patients across the UK. Design Cross-sectional, self-administered, predominantly closed-question, electronic, online survey.

Setting Institutions providing adult critical care services identified from national databases.

Participants Multiprofessional critical care clinicians delivering services at each site.

Results Responses from 176 UK hospital sites were included (176/242, 72.7\%). Inpatient recovery and follow-up services were present at 127/176 (72.2\%) sites, adopting multiple formats of delivery and primarily delivered by nurses ( $n=115 / 127,90.6 \%)$. Outpatient services ran at 130 sites (73.9\%), predominantly as outpatient clinics. Most services $(n=108 / 130$, $83.1 \%$ ) were co-delivered by two or more healthcare professionals, typically nurse/intensive care unit (ICU) physician ( $n=29 / 130,22.3 \%$ ) or nurse/ICU physician/ physiotherapist ( $n=19 / 130,14.6 \%)$ teams. Clinical psychology was most frequently lacking from inpatient or outpatient services. Lack of funding was consistently the primary barrier to service provision, with other barriers including logistical and service prioritisation factors indicating that infrastructure and profile for services remain inadequate. Posthospital discharge physical rehabilitation programmes were relatively few $(n=31 / 176$, $17.6 \%$ ), but peer support services were available in nearly half of responding institutions ( $n=85 / 176,48.3 \%)$. The effects of the COVID-19 pandemic resulted in either increasing, decreasing or reformatting service provision. Future plans for long-term service transformation focus on expansion of current, and establishment of new, outpatient services.

Conclusion Overall, these data demonstrate a proliferation of recovery, follow-up and rehabilitation services for critically ill adults in the past decade across the UK, although service gaps remain suggesting further work is required for guideline implementation. Findings can be used to enhance survivorship for critically ill adults, inform policymakers and commissioners, and provide comparative data and experiential insights for clinicians
Strengths and limitations of this study

- This is the largest and most comprehensive survey of post-critical illness recovery, rehabilitation and follow-up services available across the UK.

- This survey builds on the previous work by examining additional stages of the survivorship continuum, as well as a greater range of services.

- Our response rate achieved a representative sample of target sites, which were identified from established national registries, and with multiprofessional clinicians providing data.

- Limited data on non-responders preclude comparison with responders to detect response bias.

- Acquiring one survey response per site, regardless of number, size or specialty of intensive care units at that site may have limited detection of bespoke differences in local service delivery.

designing models of care in international healthcare jurisdictions.

\section{INTRODUCTION}

Survivorship following critical illness is characterised by varied, long-term impairments and disability that influence the quality and quantity of an individual patient's recovery. Follow-up of survivors, and other services such as multiprofessional rehabilitation, may shape recovery experiences by promoting restoration of health through identifying and appropriately managing unmet health needs associated with postintensive care syndrome. ${ }^{12}$ International reports indicate increasing development of follow-up services of varying structure, format and content ${ }^{3-9}$; however, prevalence data demonstrate their scarcity of, ${ }^{10}$ with no consistent, standardised model of service delivery. ${ }^{2}$ 
In the UK, provision of follow-up and recovery services following critical illness are embedded in national rehabilitation guidelines published in 2009 that advocate a continuum of multiprofessional input spanning the recovery pathway from intensive care unit (ICU) admission to community stages. ${ }^{12}{ }^{13}$ Considered the 'gold standard' for patient management, a face-to-face review of patients is specifically recommended at 2-3 months after critical care discharge, including a functional reassessment and onwards referral to appropriate rehabilitation or other specialist services. ${ }^{12}$ However, a nationwide survey in 2013 reviewing implementation of these guidelines found that only $27 \%$ of UK ICUs adhered to this recommendation and only 12/176 organisations offered posthospital discharge rehabilitation programmes. ${ }^{10}$ Lack of funding was both the most frequent, and highest ranking, barrier to providing services, alongside insufficient prioritisation and insufficient personnel and other resources. ${ }^{10}$ The intervening years have witnessed increasing attention on recovery services for critically ill patients, ${ }^{14-16}$ including the role of peer support. ${ }^{17}$ Therefore, the aim of this study was to comprehensively re-survey the current provision of recovery and follow-up services for adult critically ill patients across the UK to identify unmet areas of unmet need, inform service innovation and benchmark against clinical standards.

\section{METHODS}

\section{Service identification}

The sample frame was all adult National Health Service (NHS) ICUs across the UK (England, Scotland, Wales and Northern Ireland) identified using the following two central registries: the Intensive Care National Audit and Research Centre (ICNARC) Case Mix Programme (available at https://www.icnarc.org/Our-Audit/Audits/ Cmp/About/Participation) and the Scottish Intensive Care Society Audit Group (https://www.sicsag.scot.nhs. uk/index.html). A total of 242 individual hospitals were identified from the ICUs listed in these registries.

\section{Survey development}

A cross-sectional, predominantly closed-question, online open-survey was designed by the investigators (see online supplemental file 1). Survey content was generated from collective clinical experience and expertise of the investigators using the previous survey as a foundation. ${ }^{10}$ Survey questions were sequentially ordered, iteratively refined, with single or multiple response options created for each question, and inclusion of free-text options for further relevant detail. Pilot testing was done by three independent, and one internal, critical care practitioners with specialist subject interest and experience. This process ensured content, construct, and face validity, and sensibility, to ensure (1) comprehension and interpretation of questions; (2) flow, salience, acceptability and ease of completion; (3) missing items or response options and (4) time required to complete. ${ }^{18}$ Survey content was also reviewed by members of the Faculty of Intensive Care Medicine Life After Critical Illness Working Group. After refinement and optimisation, the final version was approved by the investigators.

Survey domains were as follows: (1) demographics of critical care services; (2) services delivered on inpatient wards after ending critical care, including the transfer process from ICU; (3) outpatient services delivered following hospital discharge; (4) service relationships with other local healthcare infrastructure; (5) peer support programmes and (6) physical rehabilitation programmes. Respondents were requested to report their pre-COVID-19 pandemic service provision. The final survey question requested respondents to report any changes to existing, or development of new, services due to the pandemic.

\section{Survey distribution}

An invitation email containing the link to the online survey (hosted via Survey Monkey, https://www.surveymonkey.com/) and a Participant Information Sheet was circulated via (1) Faculty of Intensive Care Medicine membership, (2) national critical care networks across each of the four UK nations, (3) the National Institute for Health Research Critical Care National Specialty Group, (4) the ICNARC Case Mix Programme membership, (5) professional contacts of the authors and (6) related social media, that facilitated a snowballing approach to dissemination. Instructions for survey completion highlighted the need for a designated lead respondent to coordinate an accurate multiprofessional response from each site. The survey was open for completion for a period of 8 weeks (June-August 2020), and repeated circulation of the survey, including targeted approaches to nonresponders where possible, was undertaken during this period. A further 4 weeks was allowed for follow-up with sites on data queries.

\section{Patient and public involvement}

Patients were not involved in the design, conduct or reporting of this research as it was focused on surveying current clinical services. However, findings from this survey will inform white papers to be developed and reported by the Faculty of Intensive Care Medicine Life After Critical Illness Working Group which includes patient and family representation.

\section{Data management and data analysis}

Survey completion was considered indicative of informed consent for participation. Data were downloaded from the survey platform into Microsoft Excel (Microsoft Corp, Redmond, WA, USA), and stored in password-protected files and devices. ${ }^{19}$ Multiple responses for any individual hospital site were de-duplicated and amalgamated into one single response set. Respondents were contacted for missing or erroneous data, or the most complete and/or first-received response set was used as the final response option. Descriptive statistics were used to analyse 
quantitative responses including normality testing, mean scores and SD, medians and IQR, frequencies, proportions, and $95 \%$ CIs where appropriate. Summative content analysis was used for free-text comments. ${ }^{20} \mathrm{~A}$ response rate of $>70 \%$ was considered a priori to indicate a representative sample. ${ }^{18} 21$ Analyses were performed in Microsoft Excel and GraphPad Prism (V.9.0, GraphPad Software, San Diego, CA, USA).

\section{RESULTS}

\section{Responding institutions}

In total, 186/242 (76.9\%) individual hospitals registered a survey response. Ten blank responses were discounted leaving 176 hospitals included in analysis (176/242, $72.7 \%$, ); across the four UK nations, this comprised Scotland $(n=23 / 23,100.0 \%)$, Wales $(n=12 / 15,80.0 \%)$, Northern Ireland $(\mathrm{n}=7 / 9,77.8 \%)$ and England $(144 / 195$, $73.8 \%)$. Demographic data for respondent hospitals are reported in table 1 .

\section{Inpatient critical illness recovery and follow-up services}

All respondents reported processes for managing discharge handovers for patients transitioning from critical care to the ward. Data describing these handover processes are reported in online supplemental file 2 section E1. Following ICU step down, 127/176 (72.2\%) operated a targeted inpatient recovery/follow-up service, established for a median (IQR) of 10.0 (5.0-16.0) years. Twenty sites $(20 / 176,11.4 \%)$ focused solely on outreach readmission prevention. Key features of services are summarised in table 2 and online supplemental file 2 section E2. Diverse service models included bedside consultation, education of ward staff around post-ICU issues, information provision to patients and families, and multiprofessional ward rounds. Where services were available, they were primarily delivered by nurses $(\mathrm{n}=115 / 127$, $90.6 \%)$, physiotherapists $(\mathrm{n}=70 / 127,55.1 \%)$ or ICU physicians $(\mathrm{n}=47 / 127,37.0 \%)$, with clinical psychology most frequently cited as lacking $(\mathrm{n}=55 / 127,43.3 \%)$. Referrals were generated from manual patient-list triages $(\mathrm{n}=80 / 127,63.0 \%)$, automated systems $(\mathrm{n}=23 / 127$, $18.1 \%)$ or electronic patient records $(n=20 / 127,15.7 \%)$. Just over half of respondents $(\mathrm{n}=69 / 127,54.3 \%)$ used a screening tool to identify postintensive care issues (eg, anxiety and depression, post-traumatic stress disorder, physical and functional performance, delirium or psychological status). Funding for services was primarily from internal critical care funds $(\mathrm{n}=71 / 127,55.9 \%)$ and institutional health service funds $(n=45 / 127,30.6 \%)$ with other sources including organisational charities, grant funding, non-critical care departments or volunteer goodwill cover (all $<10 \%)$.

\section{Outpatient critical illness recovery and follow-up services}

Outpatient services were reported in 130 institutions $(130 / 176,73.9 \%)$ established for a median (IQR) of 9.0 (4.0-15.0) years (table 3$)$, with expanded data reporting
Table 1 Demographics of respondent hospitals

\begin{tabular}{ll}
\hline Characteristic & $\mathbf{n}(\mathbf{1 1 7 6 , \% )}$ \\
\hline Type of hospital & \\
\hline District general & $99(56.3)$ \\
\hline University teaching & $63(35.8)$ \\
\hline Specialist centre & $11(6.3)$ \\
Other & $3(1.7)$ \\
\hline Profession of survey respondent & \\
\hline Medic & $79(44.9)$ \\
\hline Nurse & $42(23.9)$ \\
\hline Physiotherapist & $21(11.9)$ \\
\hline Other† & $34(19.3)$ \\
\hline Critical care service metrics & \\
\hline Total critical care beds & 3979 \\
\hline Total ICU capability & 2382 \\
\hline Total HDU capability & 1597 \\
\hline Estimated annual ICU admissions & 116944 \\
\hline Type of critical care unitł & \\
\hline General (mixed medical and surgical) & $167(94.9)$ \\
\hline Trauma & $52(29.5)$ \\
\hline Cardiothoracic & $35(19.9)$ \\
\hline Neurological/Neurosurgery & $34(19.3)$ \\
\hline Spinal & $28(15.9)$ \\
\hline Liver & $26(14.8)$ \\
\hline Burns & $19(10.8)$ \\
\hline ECMO & $9(5.1)$ \\
\hline Other & $37(21.0)$ \\
\hline
\end{tabular}

*Other includes the following: university-affiliated and specialist combined, $\mathrm{n}=3$.

†Other includes the following: (1) profession not specified/reported, $n=26$ (eg, team lead, clinical director, ward manager); (2) various, $n=5$ (eg, clinical educator, audit lead); (3) psychologist, $n=2$ and (4) dietitian, $n=1$.

$\ddagger$ Respondents could select more than one response therefore percentage exceeds $100 \%$.

§Other denotes various specialties, for example, oncology, maxilla-facial, obstetrics and renal.

ECMO, extracorporeal membrane oxygenation; HDU, high dependency unit; ICU, intensive care unit.

in online supplemental file 2 section E3. Magnitude of outpatient caseload varied from an estimated $10-500$ new patients per year, and subsequent outpatient re-evaluations ranging from an estimated $0-350$ per year. An estimated 12000 patients receive outpatient follow-up per year (at responding institutions only, out of approximately 117000 estimated annual ICU admissions). The predominant service model was an outpatient clinical consultation lasting 30-60 min and scheduled 2-3 months following hospital discharge. Patients are consulted by the multiprofessional team all together $(\mathrm{n}=77 / 130$, $59.2 \%)$ or separately one at a time $(\mathrm{n}=42 / 130,32.3 \%)$ by clinician(s), primarily comprising nurse $(n=121 / 130$, 93.1\%), ICU physician ( $\mathrm{n}=100 / 130,76.9 \%)$ and physiotherapy $(\mathrm{n}=65 / 130,50.0 \%)$ professions. In most services $(n=108 / 130,83.1 \%)$, a combination of two, three or more, different multiprofessional clinicians ran services 
Table 2 Features of targeted inpatient recovery and followup services following critical illness

\begin{tabular}{|c|c|c|}
\hline Feature & Options & $n / 127(\%)$ \\
\hline \multirow[t]{11}{*}{$\begin{array}{l}\text { Type of service } \\
\text { provision* }^{\star}\end{array}$} & $\begin{array}{l}\text { Outreach/rapid } \\
\text { response (patient } \\
\text { outcomes) }\end{array}$ & $71(55.9)$ \\
\hline & $\begin{array}{l}\text { Engagement/education } \\
\text { of ward staff regarding: } \\
\text { post-ICU issues }\end{array}$ & $65(51.2)$ \\
\hline & Information provision & $62(48.8)$ \\
\hline & $\begin{array}{l}\text { ICU physician /AHP/ } \\
\text { nurse ward round }\end{array}$ & $47(37.0)$ \\
\hline & Family support & $36(28.3)$ \\
\hline & $\begin{array}{l}\text { Psychological } \\
\text { intervention }\end{array}$ & $36(28.3)$ \\
\hline & $\begin{array}{l}\text { Generic rehabilitation } \\
\text { assistant/care } \\
\text { coordinator }\end{array}$ & $25(19.7)$ \\
\hline & Peer support & $23(18.1)$ \\
\hline & Formal MDT meeting & $17(13.4)$ \\
\hline & $\begin{array}{l}\text { Research/academic } \\
\text { contact }\end{array}$ & $8(6.35 .4)$ \\
\hline & Other† & $15(11.8)$ \\
\hline \multirow[t]{8}{*}{ Eligibility criteria } & All patients & $72(56.7)$ \\
\hline & $\begin{array}{l}\text { Length of stay in } \\
\text { critical care } \neq\end{array}$ & $54(42.5)$ \\
\hline & Clinician/ward referral & $37(29.1)$ \\
\hline & $\begin{array}{l}\text { Days of mechanical } \\
\text { ventilation§ }\end{array}$ & $31(24.4)$ \\
\hline & $\begin{array}{l}\text { Type of therapies } \\
\text { received during critical } \\
\text { care admission }\end{array}$ & $21(16.5)$ \\
\hline & Self-referral & $14(11.0)$ \\
\hline & $\begin{array}{l}\text { Diagnosis at critical } \\
\text { care admission }\end{array}$ & $11(8.7)$ \\
\hline & Other ${ }^{\eta^{\star \star}}$ & $28(19.0)$ \\
\hline \multirow{13}{*}{$\begin{array}{l}\text { Professions } \\
\text { involved in service } \\
\text { delivery }\end{array}$} & Nurse & $115(90.6)$ \\
\hline & Physiotherapist & $70(55.1)$ \\
\hline & ICU physician & $47(37.0)$ \\
\hline & $\begin{array}{l}\text { Speech and language } \\
\text { therapist }\end{array}$ & $41(32.3)$ \\
\hline & Dietitian & $39(30.7)$ \\
\hline & Occupational therapist & 27 (21.3) \\
\hline & Pharmacist & 27 (21.3) \\
\hline & $\begin{array}{l}\text { Generic rehabilitation } \\
\text { assistant }\end{array}$ & $19(15.0)$ \\
\hline & Psychologist & $17(13.4)$ \\
\hline & Administrative support & $13(10.2)$ \\
\hline & Social worker & $8(6.3)$ \\
\hline & Psychiatrist & $5(3.9)$ \\
\hline & Other†† & $19(15.0)$ \\
\hline
\end{tabular}

Continued

\begin{tabular}{lll}
\multicolumn{2}{l}{ Table 2} & Continued \\
\hline Feature & Options & n/127 (\%) \\
\hline Key challenges & Staffing number & $104(81.9)$ \\
to delivering and & Time & $90(70.9)$ \\
sustaining services & Staffing profile & $43(33.9)$ \\
& Patient location & $25(19.7)$ \\
& Environment & $21(16.5)$ \\
& Funding & $12(9.4)$ \\
& Otherł‡ & $14(11.0)$
\end{tabular}

*99 sites reported outreach services for readmission prevention in addition to targeted recovery and follow-up services.

†Other includes the following: nurse review, $n=6$; multiprofessional input, $n=6$; patient support, $n=2$; physiotherapy input, $\mathrm{n}=1$.

$\ddagger>2$ days, $n=1 ; 3$ days, $n=6$; $>3$ days, $n=8$; 4 days, $n=1$; $>4$ days, $n=5 ;>7$ days, $n=3$.

§Any, $n=1 ; 2$ days, $n=1 ; 3$ days, $n=2 ;>3$ days, $n=4 ;>4$ days, $\mathrm{n}=5$.

ПOther includes the following: patient pathway, $n=7$; delirium, $n=7$; rehabilitation needs, $n=5$; psychological status, $n=3$; physical status, $n=3$; age, $n=2$; illness acuity level, $n=1$.

${ }^{* *}$ Patients receiving palliative care, or other specialist care/ diagnosis-related pathways, and routine postoperative patients were generally not included in services.

††Other includes the following: outreach team, $n=14$; other rehabilitation/medical healthcare professionals, $n=3$; advanced critical care practitioner and counsellor, both $\mathrm{n}=1$.

¥¥Other includes the following: staffing capacity, $n=5$; lack of service prioritisation by management, $n=3$; staff engagement with service, $n=3$; staff recruitment, $n=2$; links with primary care, resources, and appropriate service focus, all $n=1$.

AHP, Allied health professional; ICU, intensive care unit; MDT, multidisciplinary team; NHS, National Health Service.

(figure 1, online supplemental file 2 table E1). The professional discipline most frequently cited as lacking was clinical psychology $(\mathrm{n}=61 / 130,46.9 \%)$.

Clinician, and self, referrals, were the most common routes to access services. Similar numbers of services reported acceptance $(\mathrm{n}=50 / 130,38.5 \%)$, and nonacceptance $(n=48 / 130,36.9 \%)$, of referrals from outside the geographical catchment area of the primary hospital (31/130 respondents, $23.8 \%$, reported this as discretionary). Over half of services $(58.5 \%)$ used a screening tool for postintensive care issues, with a heterogenous range of outcome measures and/or tools for assessment (online supplemental file 2 table E2). Aspects of recovery addressed in follow-up consultations were diverse and comprehensive, reflecting both symptom presentation as well as onwards referrals to specialist services (table 3); nearly all included a review of the patient's ICU history $(n=123 / 130,94.6 \%)$, and for the majority, an opportunity to visit to the ICU where they had been admitted 
Table 3 Features of outpatient recovery and follow-up services

\begin{tabular}{|c|c|c|}
\hline Feature & Options & $\begin{array}{l}\text { Frequency of } \\
\text { occurrence } \\
(/ 130, n, \%)\end{array}$ \\
\hline \multirow{8}{*}{$\begin{array}{l}\text { Eligibility } \\
\text { criteria }\end{array}$} & Clinician referral & 60 (46.2) \\
\hline & Self-referral & 49 (37.7) \\
\hline & Diagnosis & 22 (16.9) \\
\hline & $\begin{array}{l}\text { Length of stay critical } \\
\text { care }^{*}\end{array}$ & $18(13.8)$ \\
\hline & $\begin{array}{l}\text { Days of mechanical } \\
\text { ventilation } \dagger\end{array}$ & $17(13.1)$ \\
\hline & Therapies received & $11(8.5)$ \\
\hline & All patients & 8 (6.2) \\
\hline & Other $\ddagger$ & $18(13.8)$ \\
\hline \multirow{8}{*}{$\begin{array}{l}\text { Process for } \\
\text { identifying } \\
\text { eligible } \\
\text { patients }\end{array}$} & $\begin{array}{l}\text { Triage of all critical care } \\
\text { discharges }\end{array}$ & $79(60.8)$ \\
\hline & Review of care records & $52(40.0)$ \\
\hline & Local database & 45 (34.6) \\
\hline & Verbal clinician referral & $37(28.5)$ \\
\hline & Automated IT process & $19(14.6)$ \\
\hline & $\begin{array}{l}\text { EPR request for clinic } \\
\text { appointment }\end{array}$ & $10(7.7)$ \\
\hline & $\begin{array}{l}\text { Blanket invitation to all } \\
\text { patients (no triage) }\end{array}$ & $9(6.9)$ \\
\hline & Other§ & $2(1.5)$ \\
\hline \multirow{4}{*}{$\begin{array}{l}\text { Process of } \\
\text { monitoring } \\
\text { patients }\end{array}$} & $\begin{array}{l}\text { Ad hoc patient list/ } \\
\text { spreadsheet }\end{array}$ & $94(72.3)$ \\
\hline & Automated process & $15(11.5)$ \\
\hline & $\begin{array}{l}\text { Electronic patient } \\
\text { record-generated list }\end{array}$ & $13(10.0)$ \\
\hline & Other database & $3(2.3)$ \\
\hline \multirow{4}{*}{$\begin{array}{l}\text { Method } \\
\text { of patient } \\
\text { contact } \\
\text { regarding } \\
\text { appointment }\end{array}$} & Postal letter & $124(95.4)$ \\
\hline & Telephone call & $88(67.7)$ \\
\hline & Text reminder & $20(15.4)$ \\
\hline & Other $ף$ & $10(7.7)$ \\
\hline \multirow{4}{*}{$\begin{array}{l}\text { Funding } \\
\text { sources for } \\
\text { outpatient } \\
\text { services }^{\star *}\end{array}$} & $\begin{array}{l}\text { Funded internally from } \\
\text { critical care funds }\end{array}$ & $65(50.0)$ \\
\hline & $\begin{array}{l}\text { National health service } \\
\text { funding }\end{array}$ & $38(29.2)$ \\
\hline & Volunteer/goodwill only & 19 (14.6) \\
\hline & $\begin{array}{l}\text { Other internal } \\
\text { institutional funding }\end{array}$ & $7(5.4)$ \\
\hline \multirow[t]{6}{*}{$\begin{array}{l}\text { Aspects of } \\
\text { consultation }\end{array}$} & $\begin{array}{l}\text { Review of ICU history } \\
\text { and ICU events }\end{array}$ & $123(94.6)$ \\
\hline & Patient visit to ICU & $114(87.7)$ \\
\hline & Assessment of sleep & $99(76.2)$ \\
\hline & $\begin{array}{l}\text { Physical function } \\
\text { assessment }\end{array}$ & $96(73.8)$ \\
\hline & $\begin{array}{l}\text { Return/review of ICU } \\
\text { diary }\end{array}$ & 94 (72.3) \\
\hline & Physiotherapy referral & $91(70.0)$ \\
\hline
\end{tabular}

Table 3 Continued

\begin{tabular}{lll}
\hline & $\begin{array}{l}\text { Frequency of } \\
\text { occurrence } \\
\text { Feature }\end{array} \quad$ Options & $(/ 130, \mathrm{n}, \%)$ \\
\hline
\end{tabular}

Psychological assessment

Clinical psychology $70(53.8)$ referral

Lifestyle/risk factor 69 (53.1)

review

$67(51.5)$

Dietitian referral

Speech and language $\quad 60(46.2)$

therapy referral

Family/caregiver needs 54 (41.5) assessment

Review of goals and $53(40.8)$

preferences of care

$50(38.5)$

Employment/

occupation review

Assessment of sexual 49 (37.7)

function

Occupational therapy $47(36.2)$

referral

Nutritional assessment 47 (36.2)

Pharmacy review/ 46 (35.4)

medicines reconciliation

Cognitive assessment 38 (29.2)

Vital signs/observations 33 (25.4)

Physical examination 33 (25.4)

Social needs $\quad 33(25.4)$

assessment

Travel assessment (eg, 31 (23.8) driving, flying)

Assessment of financial 19 (14.6) status

Occupational function $13(10.0)$ assessment

Speech and language $12(9.2)$

assessment 11 (8.5)

Psychiatric assessment

Immunisation review $10(7.7)$

GP referral/information 8 (6.2)

Other††

7 (5.4)

\begin{tabular}{|c|c|c|c|}
\hline \multirow{9}{*}{$\begin{array}{l}\text { Duration of } \\
\text { appointment }\end{array}$} & & 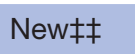 & Follow-up ${ }^{\S \S}$ \\
\hline & $<30 \min$ & $3(2.3)$ & 24 (18.5) \\
\hline & $30 \mathrm{~min}-1$ hour & 67 (51.5) & $61(46.9)$ \\
\hline & $1.0-1.5$ hours & $46(35.4)$ & 15 (11.5) \\
\hline & 1.5-2 hours & $7(5.4)$ & $2(1.5)$ \\
\hline & $2-2.5$ hours & $2(1.5)$ & $3(2.3)$ \\
\hline & 2.5-3.0 hours & $2(1.5)$ & 0 \\
\hline & $>3$ hours & $2(1.5)$ & 0 \\
\hline & Other & 0 & $13(10.0)$ \\
\hline
\end{tabular}

Continued 
Table 3 Continued

\begin{tabular}{|c|c|c|}
\hline Feature & Options & $\begin{array}{l}\text { Frequency of } \\
\text { occurrence } \\
(/ 130, n, \%)\end{array}$ \\
\hline \multirow{9}{*}{$\begin{array}{l}\text { Key } \\
\text { challenges } \\
\text { to delivering } \\
\text { and sustaining } \\
\text { services }\end{array}$} & Time & 107 (82.3) \\
\hline & Funding & $95(73.1)$ \\
\hline & Personnel & $71(54.6)$ \\
\hline & Space & $67(51.5)$ \\
\hline & $\begin{array}{l}\text { Perceived value or } \\
\text { priority }\end{array}$ & $52(40.0)$ \\
\hline & $\begin{array}{l}\text { Managerial } \\
\text { engagement }\end{array}$ & 37 (28.5) \\
\hline & $\begin{array}{l}\text { Pressure from other } \\
\text { services }\end{array}$ & $27(20.8)$ \\
\hline & Staff engagement & 15 (11.5) \\
\hline & Otherףी & $10(7.7)$ \\
\hline
\end{tabular}

* $\geq 2$ days, $n=6$; $\geq 3$ days, $n=15 ; 4$ days, $n=6$; $\geq 5$ days, $n=6 ; \geq 7$ days, $\mathrm{n}=4 ;>14$ days, $\mathrm{n}=1$.

$\dagger>24$ hours, $n=1 ; \geq 2$ days, $n=5$; $\geq 3$ days, $n=12 ; \geq 4$ days, $n=6$; $\geq 5$ days, $\mathrm{n}=7$.

fOther includes the following: illness acuity, $n=6$; postintensive care syndrome, $n=5$; delirium, $n=5$; psychological problems, $n=3$; age, $n=2$; neurological impairment and locality, both $n=1$. Short length of stay ( $<48$ hours) and/or non-ventilated patients generally not deemed eligible for follow-up.

§Other includes the following: self-referral, $n=1$, via support group, $\mathrm{n}=1$.

ๆOther includes the following: given appointment prior to hospital discharge, $n=5$; email, $n=4$; information leaflet, $n=1$.

${ }^{* *} n=1$ missing response. Respondents $(n=7)$ also commented that commissioned services for some patients, for example, trauma were available; outreach services and charity support contributed some funding and some elements of some services were unfunded.

††Other includes the following: general review, $n=3$; signposting to local services; referral to other specialties; patient/relative feedback on service; cardiac/respiratory/exercise referral, all $\mathrm{n}=1$.

$\ddagger \ddagger n=1$ missing response.

$\S \S 0$ ther includes the following: no subsequent follow-up appointment, $n=10$; no consistent follow-up appointment, $n=2$; variable duration, $\mathrm{n}=1$.

११Other includes the following: None, $n=2$; lack of administrative support and lack of referral pathways, $n=2$; lack of community services, patient engagement, insufficient patient need and current pandemic, all $n=1$.

( $\mathrm{n}=114 / 130,87.7 \%)$. Funding for services was primarily sourced from internal critical care funds $(n=65 / 130$, $50.0 \%$ ) with nearly a third underpinned by national health service-funding $(\mathrm{n}=38 / 130,29.2 \%)$, and a small proportion unfunded $(\mathrm{n}=19 / 130,14.6 \%)$.

\section{Barriers and challenges to offering recovery and follow-up services, and links with other services}

Sites without inpatient or outpatient services cited the following barriers: lack of funding ( $\mathrm{n}=35 / 46,76.1 \%)$, insufficient staff $(n=26 / 46,56.5 \%)$, lack of space/ venue $(\mathrm{n}=17 / 46,37.0 \%)$, lack of service prioritisation by management $(\mathrm{n}=17 / 46,37.0 \%)$, lack of suitably trained staff $(n=12 / 46,26.1 \%)$, resources prioritised to other patient groups/clinical areas $(\mathrm{n}=13 / 46,28.3 \%)$, lack of evidence to suggest benefit ( $n=8 / 46,17.4 \%)$, insufficient patient numbers to justify $(\mathrm{n}=5 / 46,10.9 \%)$ and uncertainty regarding content to include in a service $(n=3 / 46$, $6.5 \%$ ). Many of these resonated as challenges to service delivery and maintenance reported by those with existing services (tables 2 and 3), in particular issues of staffing, funding, and service prioritisation.

Three-quarters of respondents (133/176, 75.6\%) reported links between their own and similar services in neighbouring institutions (online supplemental file 2 section E4); categories fell broadly into two themes reflecting informal knowledge, practice, and service reciprocity, and formal referral pathway access and coordination. Links with primary care or community interface services were less frequent $(87 / 176,49.4 \%)$, with examples centring on either direct referral into services, or varied forms of engagement with primary care physicians.

\section{Peer support after critical illness}

Peer support services for patients and families were available in nearly half of responding institutions $(n=85 / 176$, 48.3\%; online supplemental file 2 section E5), predominantly as community or hospital-based support group meetings $(n=57 / 85,67.1 \%)$. Other formats included peer support groups based within ICU follow-up clinics $(\mathrm{n}=11 / 85,12.9 \%)$ or within ICU $(\mathrm{n}=5 / 85,5.9 \%)$, psychologist-led outpatient groups $(n=4 / 85,4.7 \%)$ or affiliation with ICU charity-led support groups $(n=3 / 85$, $3.5 \%)$.

Peer support varied between informal meetings $(\mathrm{n}=35 / 85,41.2 \%)$, facilitated discussion $(\mathrm{n}=20 / 85$, $23.5 \%)$ or a structured agenda of talks and presentations $(\mathrm{n}=9 / 85,10.6 \%)$. Twelve respondents $(12 / 85$, $14.1 \%$ ) reported a 'drop-in' structure, and a further $9 / 85(10.6 \%)$ reported a mixed, flexible approach. On average, sessions (of any format or structure) were held a median (IQR) of 4.5 (4.0-9.0) times per year, although absolute frequency ranged largely (minimum-maximum $1.0-52.0$ per year). Participant attendance was a median (IQR) of 10.0 (6.0-15.0) former patients and 6.0 (5.0$10.0)$ caregivers. Staff input was multiprofessional; critical care nursing staff being involved in nearly all services $(\mathrm{n}=81 / 85$, 95.3\%), with ICU physician $(\mathrm{n}=40 / 85$, $47.1 \%)$ and allied health professional $(\mathrm{n}=39 / 85,45.9 \%)$ staff involved in nearly half, and psychologists in $17 / 85$ $(20.0 \%)$. Most services were not affiliated to any formal networks $(n=49 / 85,57.6 \%)$. Where affiliation was in place $(n=33 / 85,38.8 \%)$, this was primarily with national UK networks (ICU Steps (https://www.icusteps.org/), $\mathrm{n}=27$ and InS:PIRE (Intensive care Syndrome: Promoting Independence and Return to Employment, www.nhsggc. org.uk/inspire), $\mathrm{n}=2$ ), and the international CAIRO network (Critical and Acute Illness Recovery Organization, https://sites.google.com/umich.edu/cairo/home, $\mathrm{n}=4)$. 


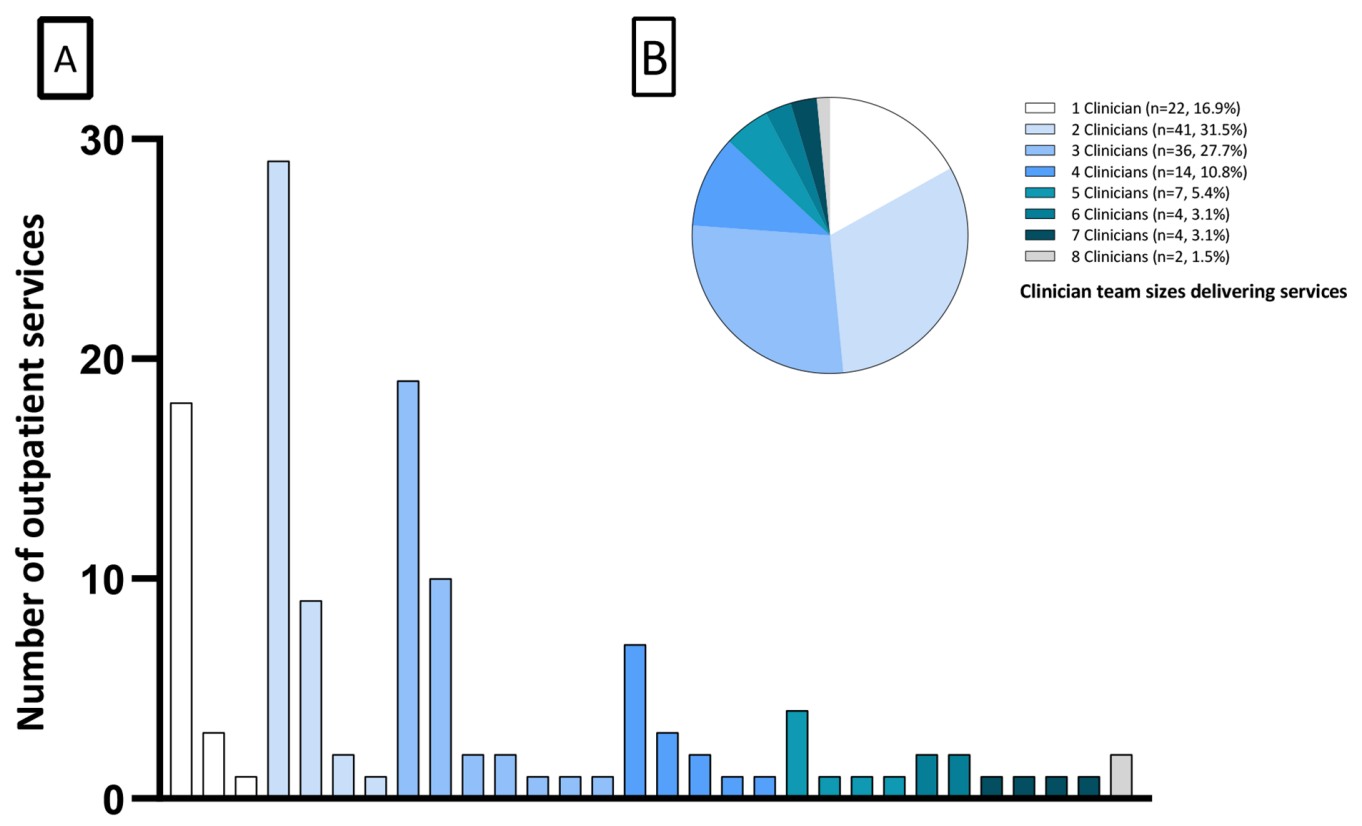

\begin{tabular}{|l|c|}
\cline { 2 - 2 } \multicolumn{1}{c|}{} & $\mathrm{n}(\%)$ \\
\hline Nurse & $121(93.1)$ \\
\hline ICU physician & $100(76.9)$ \\
\hline PT & $65(50.0)$ \\
\hline OT & $14(10.8)$ \\
\hline Psychologist & $36(27.7)$ \\
\hline Psychiatrist & $3(2.3)$ \\
\hline SLT & $9(6.9)$ \\
\hline GRA & $1(0.8)$ \\
\hline Dietitian & $11(8.5)$ \\
\hline Pharmacist & $10(7.7)$ \\
\hline GP & $1(0.8)$ \\
\hline
\end{tabular}

Composition of service

Figure 1 Composition (A) and size (B) of multiprofessional teams delivering outpatient recovery and follow-up services. (A) Bar graph depicts number of outpatient services with various multiprofessional team combinations. Detail of each corresponding profession is summarised in the table below. Total number of services $=130$. Online supplemental file 2 table E1 provides additional data on exact frequencies of occurrence of each combination. $\mathrm{N}(\%)$ detailed by each profession reports the frequency of involvement of each profession across all 130 outpatient services. $n=14$ (10.8\%) of 'other' professions involved Citizens Advice Bureau, $n=4$; volunteers, $n=2$; carers association, $n=2$; cognitive behavioural therapy, rehabilitation team, advanced critical care practitioner, patient liaison service, head injury specialist, health promotion advisor, all $\mathrm{n}=1$. Generic rehabilitation assistants are healthcare workers (some may have healthcare qualifications, but this is not essential) who offer support to qualified clinicians with carrying out various rehabilitation activities with patients. (B) Pie chart summarises the relative proportion of each team size (regardless of composition). GP, general practitioner; GRA, generic rehabilitation assistant; OT, occupational therapist; PT, physiotherapist; SLT, speech and language therapist.

\section{Posthospital discharge physical rehabilitation programmes}

Critical illness-specific posthospital discharge physical rehabilitation programmes were offered by $31 / 176$ (17.6\%) hospitals. Physiotherapists led all but one programme, either alone $(n=26 / 31,83.9 \%)$, or in combination with a nurse, exercise/sports therapist, rehabilitation medicine specialist or rehabilitation assistant (all $\mathrm{n}=1 / 31,3.2 \%$, each). One programme was led by an exercise/sports therapist. Clinicians leading programmes were either ICU-specialist $(\mathrm{n}=19 / 31,61.3 \%)$ or rehabilitation-specialist $(n=12 / 31,38.7 \%)$. Details of the structure, format and content of physical rehabilitation programmes are reported in online supplemental file 2 section E6.

\section{Future plans}

Respondents' comments about future plans for their services (within 2-5 years), in terms of instigation, development, or expansion were themed into categories (table 4). The main two themes centred on expansion of current, and establishment of new, outpatient services.

\section{Impact of the COVID-19 pandemic}

Nearly all respondents $(\mathrm{n}=162 / 176,92.0 \%)$ described the impact of the COVID-19 pandemic on services. Themes characterising these effects (and frequency of occurrence) were as follows: (1) existing service capacity/activity increased or decreased $(\mathrm{n}=88 / 162$, $54.3 \%)$, (2) existing service changed to telephone or 
Table 4 Themes characterising future plans for service development in next 2-5 years

\begin{tabular}{ll}
\hline Theme & $\begin{array}{l}\text { Frequency of occurrence } \\
(\text { /176) (n (\%)) }\end{array}$ \\
\hline $\begin{array}{l}\text { Expand current outpatient } \\
\text { services }\end{array}$ & $46(26.1)$ \\
$\begin{array}{l}\text { Start new outpatient service } \\
\text { Start new psychology service }\end{array}$ & $23(22.7)$ \\
$\begin{array}{l}\text { Expand current inpatient } \\
\text { services }\end{array}$ & $23(13.1)$ \\
$\begin{array}{l}\text { Start new inpatient service } \\
\text { Start new exercise rehabilitation }\end{array}$ & $13(10.4)$ \\
programme & $13(7.4)$ \\
$\begin{array}{l}\text { Maintain current services } \\
\text { Establish new pathways with } \\
\text { rehabilitation and specialist } \\
\text { services }\end{array}$ & $4(2.3)$ \\
\begin{tabular}{l} 
Nil specified \\
\hline
\end{tabular} & $46(26.7)$ \\
\hline
\end{tabular}

virtual $(n=74 / 162,45.7 \%)$, (3) new services implemented (phone-based, face-to-face, virtual or exercise) $(\mathrm{n}=57 / 162,35.2 \%)$, (4) applying for funding/new service $(n=44 / 162,27.2 \%),(5)$ existing service increased in frequency $(n=20 / 162,12.3 \%),(6)$ follow-up combined with respiratory medicine services $(n=20 / 162,12.3 \%)$, (7) no change $(n=17 / 162,10.5 \%)$, (8) shortened interval between review appointments $(n=11 / 162,6.8 \%)$, (9) addition of psychologist to service $(n=6 / 162,3.7 \%)$ and (10) research about follow-up initiated $(n=1 / 162,0.6 \%)$. Full details of respondents' narrative comments are reported in online supplemental file 2 section E7.

\section{DISCUSSION}

Findings from this comprehensive national survey characterise the continuum of multiprofessional recovery, follow-up and rehabilitation services currently provided for adult critically ill patients across the UK. Ward-based follow-up is highly prevalent, and a remarkable expansion of outpatient follow-up services is evident, while posthospital discharge physical rehabilitation programmes remain relatively low in number. Peer support services available in nearly half of sites support its importance for contributing to survivorship. Lack of funding commonly precluded service provision, and logistical and prioritisation barriers indicate that infrastructure and profile for services remains inadequate.

\section{Interpretation of the findings}

More than $70 \%$ of sites provided targeted longitudinal follow-up support to patients on the wards following ICU discharge with more than half incorporating screening for postintensive care syndrome. This is in keeping with recommended practice, ${ }^{12}$ and signifies a practice of early identification and management of problems as well as onwards recovery planning. Comparative data on prevalence of inpatient recovery services are limited; one smaller previous survey reported only around one-third of sites were guideline-adherent on ward-based input following critical illness. ${ }^{22}$

Increased prevalence of outpatient services at $74 \%$ of institutions, compared with $27 \%$ previously, ${ }^{10}$ is striking, and vastly exceeds international counterparts. ${ }^{11}$ Underlying factors behind this considerable growth are unclear, but greater appreciation of the long-term consequences of critical illness from within the clinical community could be speculated given that half of services were funded via internal critical care sources, many were delivered within existing roles without dedicated additional time, and clinician referral to services surpassed objective criteria. Scheduling of follow-up was also adherent with national recommendations. ${ }^{12}$ However, uniprofessional service delivery by nursing staff prevailed in the outpatient context despite the empirical value of many other disciplines, and even though representation from clinical psychology doubled in outpatient compared with inpatient services, this was the most frequently reported missing profession from both. This emphasises both the need for investment in personnel, and the urgency of addressing psychological morbidity in survivors, ${ }^{23-25}$ which can influence engagement with other aspects of recovery, and contribute to hospital readmission. ${ }^{26}$ Likewise, occupational therapy is another example of a key profession that would benefit from greater prevalence within services compared with the levels seen in the current findings, especially in the context of long-term cognitive impairment in critical illness survivors, ${ }^{27-29}$ and the challenges of returning to work in this patient population. ${ }^{30-33}$

Engagement with primary care reduced from inpatient to outpatient stages of management. Partnership with primary care is key to optimising quality of critical illness recovery ${ }^{34}$; qualitative exploration of unplanned hospital readmission in ICU survivors highlights many contributing themes that primary care clinicians would be ideally placed to support during recovery, for example, multimorbidity, polypharmacy, inadequate social support and challenges with specialist equipment. ${ }^{26}{ }^{35}$ Improving information provision on patients' ICU admissions and their consequences could be a simple yet effective and valued strategy to start, ${ }^{36} 37$ especially where primary care physicians may see relatively few post-ICU patients. Utilising remote, virtual platforms may facilitate this happening in person to complement written or electronic forms. Furthermore, advocating a routine appointment for postintensive care patients with their primary care clinician to review status early in the community stage of recovery; this could be held jointly with a post-ICU follow-up appointment for efficient shared clinical management and learning.

Posthospital discharge physical rehabilitation programmes also increased since last surveyed. That this increase is much more modest (from $7 \%$ to $18 \%$ ) may be multifactorial, but one possibility is the relative 'burden' 
of leading the delivery of such services by only one profession, namely physiotherapy_lack of sufficient staff features highly as a barrier in the current dataset. Broadly, the structure, format and content, of delivery of physical rehabilitation programmes mirrored previously reported findings, although two-thirds of programmes still utilised referrals to other bespoke rehabilitation programmes for example, pulmonary and cardiac, to manage unmet need even though these may not cater optimally for patients following critical illness. ${ }^{10}$ The limited overall availability of these rehabilitation services speaks to the need to consider alternative strategies to deliver therapeutic interventions. One option is to consider home-based services, which may be essential for those patients where mobility limitations preclude physical attendance at other venues, as well as those in rural areas, with social isolation, or relatively less caregiver support. The impact of the COVID-19 pandemic has seen an exponential rise in diverse models of care with greater use of virtual platforms that could be investigated further in the future to ensure maximum inclusivity of patients into rehabilitation programmes.

Peer support benefits patients, relatives, and staff during survivorship. ${ }^{15} 38{ }^{39}$ Six models have been described ${ }^{17}$; our data indicate a predominance of community-based peer support with no evidence for online delivery, although this may have evolved in the interim due to pandemic restrictions to physical in-person meeting. Barriers (eg, non-attendance, access to skilled facilitators and bureaucratic limitations) and enablers (eg, motivated interprofessional clinicians, patient and family volunteers, links to ICU follow-up clinics) to peer support services have been previously explored through focus group inquiry with clinicians. ${ }^{14} 17$ As peer support continues to embed within the armamentarium of postcritical illness recovery, including for patients surviving post-COVID- $19^{40}$, our data can be used to support the emergence of other models of delivery within the UK setting, with reference to these barriers and enablers to ensure individual participant preferences for mode of engagement with peer support are met.

Lack of funding most often precluded delivery of critical illness recovery and follow-up services, followed by availability of sufficient staff; these, and other findings on reported barriers, closely mirror previous data. ${ }^{10} \mathrm{~A}$ key issue affecting funding and deliverability is disparity between commissioning processes, often at national and local level respectively for inpatient and outpatient critical care services, that currently do not mandate adherence to the national guidelines. This disconnect fails to reflect the continuum over which recovery occurs from ICU admission to discharge home, and the attainment of individualised goals of recovery. Reliance on bespoke local commissioning applications to source funding therefore directly affects equity of access to critical care outpatient services. Key to application success are the strength of national guidelines, quality standards, patient/caregiver value, and the observation from care quality commissioners that inpatient services are impacted positively by outpatient follow-up. However, these empirical-reported benefits are often insufficient to secure funding, as reflected in this survey, because they are frequently countered by demands for evidence to demonstrate clinical and cost effectiveness; at present neither follow-up clinics or posthospital discharge physical rehabilitation programmes are supported by meta-analysis data, ${ }^{21}$ and there is an absence of consensus on the most appropriate metric to reflect 'success'. Evidence-gaps exist around the optimum version of either modality and the service-user voice is often missing in shaping research. ${ }^{15}$ Reliance on internal funding sources to deliver services results in the disparity in workforce composition seen in our findings. In the future, standardising data collection across services may serve to build evidence around the impact on patient outcomes.

How much the COVID-19 pandemic influences the current landscape of critical illness recovery, follow-up, and rehabilitation services, in the long-term remains to be seen. ${ }^{42}{ }^{43}$ Our findings indicated both 'positive' (eg, service expansion, addition of professional specialties) and 'negative' (eg, lack of resources, loss of physical in-person contact) impacts. We also detected a signal towards service digitisation, although this would require careful management to prevent issues such as digital poverty and literacy from limiting access. In the UK, postCOVID-19 follow-up clinics are underpinned by largescale national funding, and aim to address short-term and long-term sequelae affecting patients, ${ }^{44}$ but there are also data reporting international efforts, ${ }^{45}$ as well as empirical reports of local service development. We posit that the current data, detailing existing national services at a granular level, may be informative for future commissioning and policy-makers in directing resources towards services for all patients recovering from critical illness, irrespective of causal illness or injury, to ensure evidence-based provision of care. A blended payment model for critical care services, incorporating an outpatient tariff within the outcome element would be transformational. This would provide financial resources for all ICUs to include post-ICU discharge services (whereas existing funding is limited to the ICU period), enabling the standardisation and improvement in the equity of access of services for patients across all four nations.

\section{Critique of the method}

This study benefits from a number of strengths. Sampling was through two national registries, and survey design was rigorous and comprehensive, including external pilot testing. The inclusion of $i n$-hospital services increases the value of this dataset that now provides detailed characterisation on available services across the continuum of critical illness recovery. Survey platform functionality was maximised to mitigate respondent burden or fatigue. ${ }^{46}$ Survey dissemination adopted multiple methods and respondents represented a wide range of professions. This approach facilitated a high response rate exceeding 
our a priori threshold for representativeness, with minimal missing data.

We encouraged a coordinated multiprofessional response from each institution anticipating enhanced accuracy of data. However, any limitation in availability or cooperation of colleagues could hypothetically have impacted the quality and reliability of responses. Furthermore, limited data on non-responders precluded comparison with responders to detect presence of any response bias. ${ }^{21}{ }^{47}$ For pragmatic purposes, we sought one survey response per hospital, regardless of the number, size or specialty of ICUs at that hospital. However, some bespoke differences may exist in recovery, rehabilitation and follow-up services according to ICU specialty that were not detectable in this survey. Where more than one unique hospital was part of a single overarching healthcare provider, we still required an individual survey response per hospital to account for potential interhospital differences in services.

Our data reflect UK NHS provision (as of mid-2020), potentially impacting extrapolation of findings to other healthcare jurisdictions. UK national guidelines offer a valuable scaffold to guide patient management. However, the granular, multicentre, national-level data clearly demonstrate a wide range of recovery and follow-up services of varying structure, format, content, staffing, and delivery, and from a diverse population of hospitals. As such, clinicians from other international healthcare settings could consider elements for potential adaptation and translation into local services. In the future, international consensus from professional organisations around the key components of postcritical care services would be beneficial.

\section{CONCLUSION}

This study provides a comprehensive snapshot of the UK landscape of post critical illness recovery, follow-up and rehabilitation services, including an indication of the impact of pandemic circumstances. Service sustainability will require improved referral pathways, enhanced partnership with primary care, greater medical engagement and adoption of national standards. These data complement national and international efforts to optimise quality of care and outcomes of survivors of critical illness.

\footnotetext{
Author affiliations

${ }^{1}$ Wellcome-Wolfson Institute for Experimental Medicine, Queen's University Belfast, Belfast, UK

${ }^{2}$ Lane Fox Clinical Respiratory Physiology Research Centre, Guy's and St Thomas' NHS Foundation Trust, London, UK

${ }^{3}$ Centre for Human and Applied Physiological Sciences, King's College London, London, UK

${ }^{4}$ Department of Physiotherapy, The University of Melbourne, Melbourne, Victoria, Australia

${ }^{5}$ Department of Anaesthesia \& Critical Care, Royal Infirmary of Edinburgh,

Edinburgh, UK

${ }^{6}$ Ed Major Critical Care Unit, Morriston Hospital, Swansea, UK

${ }^{7} \mathrm{NHS}$ Greater Glasgow and Clyde, Glasgow, UK

${ }^{8}$ School of Medicine, Dentistry, and Nursing, University of Glasgow, Glasgow, UK
}

${ }^{9}$ The Healthcare Improvement Studies (THIS) Institute, University of Cambridge, Cambridge, UK

${ }^{10}$ Department of Critical Care, Belfast Health and Social Care Trust, Belfast, UK ${ }^{11}$ Department of Critical Care, Guy's and St.Thomas' NHS Foundation Trust, London, UK

${ }^{12}$ Department of Intensive Care and Anaesthetics, Royal Berkshire Hospital, Reading, UK

${ }^{13}$ Department of Critical Care Medicine, Royal Infirmary of Edinburgh, Edinburgh, UK

Twitter Bronwen Connolly @bronwenconnolly, Ceri Battle @ceribattle and Joanne McPeake @jomcpeake22

Acknowledgements The authors thank members of the Faculty of Intensive Care Medicine Executive, including Daniel Waeland, James Goodwin and Anna Ripley, for their support, as well as the NIHR Critical Care National Specialty Group, Intensive Care National Audit and Research Centre, the Scottish Intensive Care Society and the Welsh Intensive Care Society, for assistance in survey circulation. We acknowledge and thank our three independent colleagues, Professor Leanne Aitken, Dr Eve Corner and Dr Ryan Haines, as well as Ms Penelope Firshman (FICM Life After Critical Illness Working Group) who formally piloted the survey and gave feedback. Finally, we are hugely grateful to all respondents for their time and motivation in contributing to this study with their responses, particularly in the challenging pandemic-related clinical circumstances under which all were working at the time of participation.

Collaborators The Faculty of Intensive Care Medicine Life After Critical IIIness Working Group comprises: Carl Waldmann (Chair), Joel Meyer (Deputy Chair), Andrew Slack (Deputy Chair), Greg Barton, Anthony Bastin, Danielle Bear, Suzanne Bench, Martin Davies, Andrew Ferguson, Penny Firshman, Melanie Gager, Julie Highfield, Sarah Linford, Joanne McPeake, Judith Merriweather, Jack Parry-Jones, Margaret Phillips, Tara Quasim, Helen Sanger, Gordon Sturmey, Dorothy Wade, Elizabeth Wilson.

Contributors $\mathrm{BC}, \mathrm{AS}, \mathrm{CW}$ and JM conceived and designed the study. $\mathrm{BC}$ drafted an initial survey version, and all authors (BC, RM-C, CA, CB, JM, TQ, JS, AS, CW, EW and $\mathrm{JM}$ ) contributed to iteration and refinement in survey content and design. $\mathrm{BC}$, $\mathrm{CA}, \mathrm{CB}, \mathrm{EW}$, JS and CW facilitated survey dissemination via established networks. $\mathrm{BC}$ was responsible for overall data acquisition via the online survey platform. $\mathrm{BC}$ and $\mathrm{RMC}$ analysed the data. $\mathrm{BC}$ and JM interpreted the data and agreed data reporting. $B C$ drafted and revised manuscript versions, and all authors (BC, RM-C $\mathrm{CA}, \mathrm{CB}, \mathrm{JM}, \mathrm{TQ}, \mathrm{JS}, \mathrm{AS}, \mathrm{CW}, \mathrm{EW}$ and $\mathrm{JM}$ ) agreed the final manuscript version for submission.

Funding This research received no specific grant from any funding agency in the public, commercial, or not-forprofit sectors. JM is funded via a Fellowship from The Healthcare Improvement Studies (THIS) Institute, University of Cambridge, Cambridge, UK (PD-2019-02-16).

Competing interests None declared.

Patient consent for publication Not required.

Ethics approval The study was approved by King's College London Research Ethics Committee (MRA-19/20-17855), and is reported in keeping with the Checklist for Reporting Results of Internet E-Surveys (CHERRIES).

Provenance and peer review Not commissioned; externally peer reviewed.

Data availability statement No data are available. Data are not publicly available for confidentiality reasons, however all data are reported.

Supplemental material This content has been supplied by the author(s). It has not been vetted by BMJ Publishing Group Limited (BMJ) and may not have been peer-reviewed. Any opinions or recommendations discussed are solely those of the author(s) and are not endorsed by BMJ. BMJ disclaims all liability and responsibility arising from any reliance placed on the content. Where the content includes any translated material, BMJ does not warrant the accuracy and reliability of the translations (including but not limited to local regulations, clinical guidelines, terminology, drug names and drug dosages), and is not responsible for any error and/or omissions arising from translation and adaptation or otherwise.

Open access This is an open access article distributed in accordance with the Creative Commons Attribution Non Commercial (CC BY-NC 4.0) license, which permits others to distribute, remix, adapt, build upon this work non-commercially, and license their derivative works on different terms, provided the original work is properly cited, appropriate credit is given, any changes made indicated, and the use is non-commercial. See: http://creativecommons.org/licenses/by-nc/4.0/. 


\section{ORCID iDs}

Bronwen Connolly http://orcid.org/0000-0002-5676-5497

Joanne McPeake http://orcid.org/0000-0001-8206-6801

\section{REFERENCES}

1 Needham DM, Davidson J, Cohen $\mathrm{H}$, et al. Improving long-term outcomes after discharge from intensive care unit: report from a stakeholders' conference. Crit Care Med 2012;40:502-9.

2 Schofield-Robinson OJ, Lewis SR, Smith AF, et al. Follow-up services for improving long-term outcomes in intensive care unit (ICU) survivors. Cochrane Database Syst Rev 2018;2018.

3 Cuthbertson $\mathrm{BH}$, Rattray J, Campbell MK, et al. The practical study of nurse led, intensive care follow-up programmes for improving long term outcomes from critical illness: a pragmatic randomised controlled trial. BMJ 2009;339:b3723.

4 Fonsmark L, Rosendahl-Nielsen M. Experience from multidisciplinary follow-up on critically ill patients treated in an intensive care unit. Dan Med J 2015;62:A5062.

5 Bakhru RN, Davidson JF, Bookstaver RE, et al. Implementation of an ICU recovery clinic at a tertiary care academic center. Crit Care Explor 2019;1:e0034.

6 Sevin CM, Bloom SL, Jackson JC, et al. Comprehensive care of ICU survivors: development and implementation of an ICU recovery center. J Crit Care 2018;46:141-8.

7 Khan BA, Lasiter S, Boustani MA. Ce: critical care recovery center: an innovative collaborative care model for ICU survivors. Am J Nurs 2015;115:24-31.

8 Kvåle R, Ulvik A, Flaatten H. Follow-up after intensive care: a single center study. Intensive Care Med 2003;29:2149-56.

9 Samuelson KAM, Corrigan I. A nurse-led intensive care after-care programme - development, experiences and preliminary evaluation. Nurs Crit Care 2009:14:254-63.

10 Connolly B, Douiri A, Steier J, et al. A UK survey of rehabilitation following critical illness: implementation of NICE clinical guidance 83 (CG83) following hospital discharge. BMJ Open 2014;4:e004963.

11 Cook K, Bartholdy R, Raven M, et al. A national survey of intensive care follow-up clinics in Australia. Aust Crit Care 2020;33:533-7.

12 NICE. Rehabilitation after critical illness. NICE clinical guideline 83. London, UK: National Institute for Health and Care Excellence, 2009. http://www.nice.org.uk/guidance/cg83

13 NICE. Rehabilitation after critical illness in adults. quality standard QS158. London, UK: National Institute for Health and Care Excellence, 2017. https://www.nice.org.uk/guidance/qs158/chapter/ About-this-quality-standard

14 Haines KJ, McPeake J, Hibbert E, et al. Enablers and barriers to implementing ICU follow-up clinics and peer support groups following critical illness: the thrive collaboratives. Crit Care Med 2019;47:1194-200.

15 McPeake J, Boehm LM, Hibbert E, et al. Key components of ICU recovery programs: what did patients report provided benefit? Crit Care Explor 2020;2:e0088.

16 McPhee JS. Muscle weakness and fatigability after treatment in the ICU. Crit Care Med 2013;41:345-6.

17 McPeake J, Hirshberg EL, Christie LM, et al. Models of peer support to remediate post-intensive care syndrome: a report developed by the society of critical care medicine thrive international peer support collaborative. Crit Care Med 2019;47:e21-7.

18 Burns KEA, Duffett M, Kho ME, et al. A guide for the design and conduct of self-administered surveys of clinicians. CMAJ 2008;179:245-52.

19 Eysenbach G. Improving the quality of web surveys: the checklist for reporting results of Internet E-surveys (cherries). J Med Internet Res 2004;6:e34.

20 Hsieh H-F, Shannon SE. Three approaches to qualitative content analysis. Qual Health Res 2005;15:1277-88.

21 Rubenfeld GD. Surveys: an introduction. Respir Care 2004;49:1181-5.

22 Berry A, Cutler LR, Himsworth A. National survey of rehabilitation after critical illness. J Intensive Care Soc 2013;14:334-9.

23 Hopkins RO, Weaver LK, Collingridge D, et al. Two-year cognitive, emotional, and quality-of-life outcomes in acute respiratory distress syndrome. Am J Respir Crit Care Med 2005;171:340-7.
24 Nikayin S, Rabiee A, Hashem MD, et al. Anxiety symptoms in survivors of critical illness: a systematic review and meta-analysis. Gen Hosp Psychiatry 2016;43:23-9.

25 Rabiee A, Nikayin S, Hashem MD, et al. Depressive symptoms after critical illness: a systematic review and meta-analysis. Crit Care Med 2016;44:1744-53.

26 Donaghy E, Salisbury L, Lone NI, et al. Unplanned early hospital readmission among critical care survivors: a mixed methods study of patients and carers. BMJ Qual Saf 2018;27:915-27.

27 Geense WW, Zegers M, Peters MAA, et al. New physical, mental, and cognitive problems 1 year after ICU admission: a prospective multicenter study. Am J Respir Crit Care Med 2021;203:1512-21.

28 Nelliot A, Dinglas VD, O'Toole J, et al. Acute respiratory failure survivors' physical, cognitive, and mental health outcomes: quantitative measures versus semistructured interviews. Ann Am Thorac Soc 2019;16:731-7.

29 Pandharipande PP, Girard TD, Jackson JC, et al. Long-term cognitive impairment after critical illness. N Engl J Med 2013;369:1306-16.

30 Kamdar BB, Suri R, Suchyta MR, et al. Return to work after critical illness: a systematic review and meta-analysis. Thorax 2020;75:17-27.

31 McPeake J, Mikkelsen ME, Quasim T, et al. Return to employment after critical illness and its association with psychosocial outcomes. a systematic review and meta-analysis. Ann Am Thorac Soc 2019;16:1304-11.

32 Su H, Hopkins RO, Kamdar BB, et al. Association of imbalance between job workload and functional ability with return to work in ARDS survivors. Thorax 2021. doi:10.1136/thoraxjnl-2020-216586. [Epub ahead of print: 29 Apr 2021]

$33 \mathrm{Su} \mathrm{H}$, Thompson HJ, May S, et al. Association of job characteristics and functional impairments on return to work after ARDS. Chest 2021;160:509-18.

34 Admon AJ, Tipirneni R, Prescott HC. A framework for improving post-critical illness recovery through primary care. Lancet Respir Med 2019;7:562-4

35 Turnbull AJ, Donaghy E, Salisbury L, et al. Polypharmacy and emergency readmission to hospital after critical illness: a populationlevel cohort study. Br J Anaesth 2021;126:415-22.

36 Bench S, Cornish J, Xyrichis A. Intensive care discharge summaries for general practice staff: a focus group study. Br J Gen Pract 2016;66:e904-12.

37 Daruwalla F, Lamb FJ, Mearns CA. Quality and value of intensive care discharge summaries for general practitioners. Crit Care 2012;16:P520.

38 Groves J, Cahill J, Sturmey G, et al. Patient support groups: a survey of United Kingdom practice, purpose and performance. J Intensive Care Soc 2020;188:175114372095201.

39 McPeake J, Iwashyna TJ, Boehm LM, et al. Benefits of peer support for intensive care unit survivors: sharing experiences, care debriefing, and altruism. Am J Crit Care 2021;30:145-9.

40 Hope AA, Johnson AA, McPeake J, et al. Establishing a peer support program for survivors of COVID-19: a report from the critical and acute illness recovery organization. Am J Crit Care 2021;30:150-4.

41 Connolly B, Salisbury L, O'Neill B, et al. Exercise rehabilitation following intensive care unit discharge for recovery from critical illness. Cochrane Database Syst Rev 2015;113.

42 NICE guideline [NG188]. COVID-19 rapid guideline: managing the long-term effects of COVID-19, 2020. Available: https://www.nice. org.uk/guidance/ng188

43 Prescott HC. Outcomes for patients following hospitalization for COVID-19. JAMA 2021;325:1511-2.

44 England NHS. NHS launches 40 'long COVID' clinics to tackle persistent symptoms, 2020. Available: https://www.england.nhs.uk/ 2020/11/nhs-launches-40-long-covid-clinics-to-tackle-persistentsymptoms/

45 Writing Committee for the COMEBAC Study Group, Morin L, Savale $\mathrm{L}$, et al. Four-month clinical status of a cohort of patients after hospitalization for COVID-19. JAMA 2021;325:1525-1534.

46 Lavrakas P. Encyclopedia of survey research methods. SAGE, 2008.

47 Burkell J. The dilemma of survey nonresponse. Libr Inf Sci Res 2003;25:239-63. 